\title{
Referential Success of Set Referring Expressions with Fuzzy Properties
}

\author{
Nicolás Marín, Gustavo Rivas-Gervilla, and Daniel Sánchez \\ Dept. Computer Science and A.I., University of Granada, Spain \\ nicmedecsai.ugr.es
}

\begin{abstract}
We introduce the properties to be satisfied by measures of referential success of set referring expressions with fuzzy properties. We define families of measures on the basis of $\mathrm{k}$ specificity measures and we illustrate some of them with a toy example.
\end{abstract}

\section{Introduction}

The classical referring expression generation (REG) problem intends to determine a noun phrase which univocally identify an object in a collection. From a knowledge representation perspective, the problem is to determine (if possible) a collection of object properties that can be employed in the noun phrase for the abovementioned purpose (van Deemter, 2016).

The most usual version of the REG problem can be formalized as follows: given a context formed by a collection of objects $O$ and a set of properties $P$ of objects in $O$, determine a subset $r e \subseteq P$ such that

$$
\bigcap_{p_{i} \in r e}\left[\left[p_{i}\right]\right]=\{o\}
$$

where $\left[\left[p_{i}\right]\right]$ is the set of objects that satisfy $p_{i}$. This formalization assumes that properties in $r e$ are combined conjunctively; other logical combinations are possible (van Deemter, 2016).

There are many different extensions of the classical REG problem. One such extension is that of referring to sets (Krahmer and van Deemter, 2012; van Deemter, 2016), where the objective is to generate a referring expression able to identify an distinguish a subset of objects $O^{i} \subset O$, that is, to determine a subset $r e \subseteq P$ such that

$$
\bigcap_{p_{i} \in \text { re }}\left[\left[p_{i}\right]\right]=O^{i}
$$

Note that the classical problem is a particular case of set referring where $O^{i}$ is a singleton.

Another extension considers that properties in $P$ may be vague or uncertain in several respects (van Deemter, 2012). When the fulfilment of properties in $P$ is gradual in nature, they may be modelled by means of fuzzy sets (Gatt et al., 2016). Fuzzy properties are modelled by means of fuzzy sets, corresponding to membership functions of the form $p_{i}: O \rightarrow[0,1] \forall p_{i} \in P$, where 0 means "no fulfilment" and 1 means "total fulfilment", allowing intermediate degrees. Note that classical crisp properties are particular cases of fuzzy ones in which no intermediate degrees are allowed, and hence the classical REG problem is again a particular case of the REG problem with fuzzy properties.

In the aforementioned crisp versions of the problem, when Eq. (1) (resp. Eq. (2)) holds, it is said that $r e$ has referential success for $o\left(\right.$ resp. $\left.O^{i}\right)$. One problem that arises in REG with fuzzy properties is that, as the fulfilment of properties by objects is a matter of degree, so it is the referential success. Several proposals for determining the degree of referential success have been provided in the literature (Gatt et al., 2016; Marín et al., 2016; Marín et al., 2017c), some of them based on the notion of specificity introduced by R. Yager (Yager, 1982; Yager, 1990; Yager, 1992; Garmendia et al., 2003; Garmendia et al., 2006). 
In this paper we show a preliminary proposal for extending referential success measures to the case of set referring expressions with fuzzy properties.

\section{Referential Success for Individual Objects with Fuzzy Properties}

Let us assume that properties in $P$ are fuzzy and let $p_{i}(o)$ be the accomplishment degree of property $p_{i}$ for object $o$. The accuracy of the referring expression is then calculated as (Gatt et al., 2016):

$$
O_{r e}(o)=\bigotimes_{i=1}^{n} p_{i}(o)
$$

where $\otimes$ is a t-norm. Unless otherwise stated, we shall employ the minimum, that is, $O_{r e}(o)=$ $\min \left\{p_{i}(o)\right\} . O_{r e}$ can be seen as the fuzzy set induced by $r e$ on $O$.

In (Marín et al., 2016), a minimal set of properties that a referential success measure $r s(r e, o)$ must fulfill in relation to the induced $O_{r e}$ is presented:

Property 2.1 $r s\left(r e, o_{i}\right)=1$ iff $O_{r e}=\left\{o_{i}\right\}$.

Property 2.2 If $O_{r e}\left(o_{i}\right)=0$ then $r s\left(r e, o_{i}\right)=0$.

Property 2.3 If $O_{r e}(o) \leq O_{r e^{\prime}}(o) \forall o \in O \backslash\left\{o_{i}\right\}$ and $O_{r e}\left(o_{i}\right) \geq O_{r e^{\prime}}\left(o_{i}\right)$ then $r s\left(r e, o_{i}\right) \geq r s\left(r e^{\prime}, o_{i}\right)$.

Additionally, a general family of measures is provided in (Marín et al., 2016) on the basis of Yager's specificity measures, as follows:

Definition 2.1 (Marin et al., 2016) Let t be a t-norm and $S p$ be a specificity measure. The referential success measure associated to $S p$ and $t$ is defined as follows:

$\overrightarrow{R S}_{t}(S p)\left(r e, o_{i}\right)= \begin{cases}t\left(O_{r e}\left(o_{i}\right), S p\left(O_{r e}^{*}\right)\right) & \text { condmax } \\ 0 & \text { otherwise }\end{cases}$

where

$$
O_{r e}^{*}\left(o_{j}\right)=\frac{O_{r e}\left(o_{j}\right)}{\max _{o \in O} O_{r e}(o)}
$$

and condmax stands for the following condition:

$$
\max _{o \in O} O_{r e}(o)=O_{r e}\left(o_{i}\right)>0 .
$$

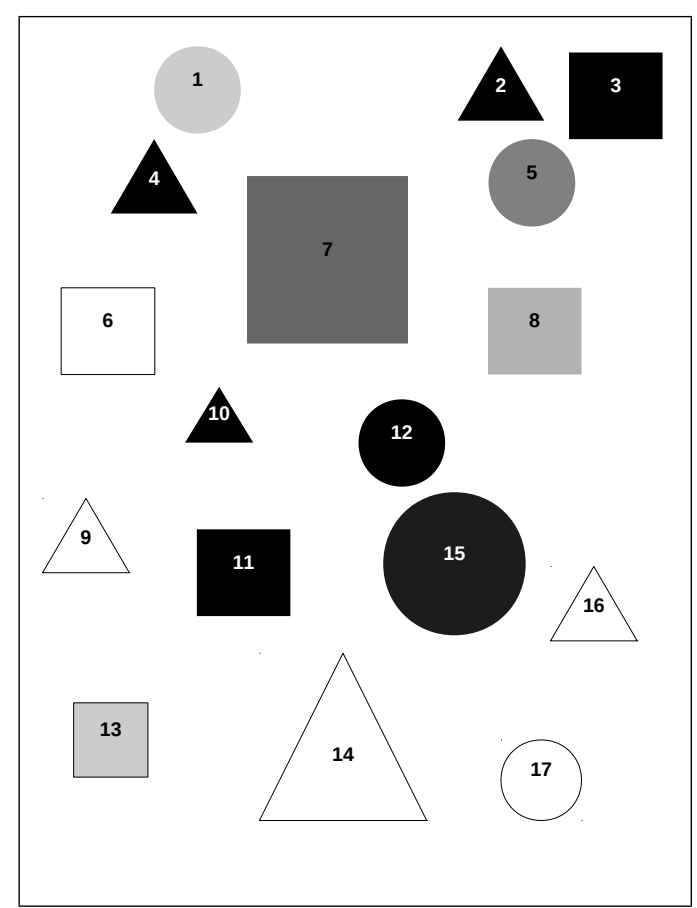

Figure 1: Example scene

\section{Referential success of Set Referring Expressions}

We propose the following minimal set of properties to be fulfilled by a referential success measure $r s$ of set referring expressions:

Property $3.1 r s\left(r e, O^{i}\right)=1$ iff $O_{r e}=O^{i}$.

Property 3.2 If $\exists o \in O^{i}$ such that $O_{r e}(o)=0$ then rs $\left(\right.$ re, $\left.O^{i}\right)=0$.

Property 3.3 If $O_{r e}(o) \leq O_{r e^{\prime}}(o) \forall o \in O \backslash O^{i}$ and $O_{r e}(o) \geq O_{r e^{\prime}}(o) \quad \forall o \in O^{i}$ then $r s\left(r e, O^{i}\right) \geq$ $r s\left(r e^{\prime}, O^{i}\right)$.

Our proposal of measures in this paper is based on the measures of $k$-specificity introduced in (Sánchez et al., 2016) and related to cardinality in (Marín et al., 2017a). The measure $S p_{k}$ indicates to which degree the cardinality of a fuzzy set is exactly $k$. Eqs. (6) to (8) show three such measures:

$$
S p_{k}^{f}(A)=\left\{\begin{array}{cl}
\frac{\prod_{i=0}^{k} a_{i}^{2}}{\prod_{i=0}^{k} a_{i}+\sum_{i=k+1}^{m} a_{i}} & a_{k}>0 \\
0 & \text { otherwise }
\end{array}\right.
$$




\begin{tabular}{l|ccccccccccccccccc} 
& 1 & 2 & 3 & 4 & 5 & 6 & 7 & 8 & 9 & 10 & 11 & 12 & 13 & 14 & 15 & 16 & 17 \\
\hline top-right & 0 & 1 & 1 & 0 & 1 & 0 & 0.1 & 0.3 & 0 & 0 & 0 & 0 & 0 & 0 & 0 & 0 & 0 \\
\hline dark & 0.3 & 1 & 1 & 1 & 0.6 & 0 & 0.7 & 0.4 & 0 & 1 & 1 & 1 & 0.3 & 0 & 0.9 & 0 & 0 \\
\hline square & 0 & 0 & 1 & 0 & 0 & 1 & 1 & 1 & 0 & 0 & 1 & 0 & 1 & 0 & 0 & 0 & 0 \\
\hline triangle & 0 & 1 & 0 & 1 & 0 & 0 & 0 & 0 & 1 & 1 & 0 & 0 & 0 & 1 & 0 & 1 & 0 \\
\hline circle & 1 & 0 & 0 & 0 & 1 & 0 & 0 & 0 & 0 & 0 & 0 & 1 & 0 & 0 & 1 & 0 & 1 \\
\hline
\end{tabular}

Table 1: Membership degrees of properties by objects in Fig. 1.

\begin{tabular}{c|c|c|c|c|c|c|}
\cline { 2 - 7 } & \multicolumn{2}{|c|}{$S p_{3}^{f}$} & \multicolumn{2}{c|}{$S p_{\Lambda, 3}$} & \multicolumn{2}{c|}{$S p_{L, 3}$} \\
\cline { 2 - 7 } & $t=\min$ & $t=$ prod & $t=\min$ & $t=\operatorname{prod}$ & $t=\min$ & $t=\operatorname{prod}$ \\
\hline Fig. 2(a) & 1 & 1 & 1 & 1 & 1 & 1 \\
\hline Fig. 2(b) & $1 / 3$ & $1 / 3$ & 0 & 0 & 0 & 0 \\
\hline Fig. 2(c) & $5 / 7$ & $5 / 7$ & 0.7 & 0.7 & 0.7 & 0.7 \\
\hline Fig. 2(d) & 0.35 & 0.245 & 0.21 & 0.147 & 0.3 & 0.21 \\
\hline
\end{tabular}

Table 2: Different referential success measures applied to scenes in Fig. 2.

$$
\begin{gathered}
S p_{\Lambda, k}(A)=a_{k}\left(a_{k}-a_{k+1}\right) \\
S p_{L, k}(A)=a_{k}-a_{k+1}
\end{gathered}
$$

We define the following family of measures of referential success:

Definition 3.1 Let t be a t-norm, let $\left|O^{i}\right|=n>0$ and $S p_{k}$ be a measure of $k$-specificity. The referential success measure associated to $S p_{k}$ and $t$ is defined as follows:

$\overrightarrow{R S}_{t}\left(S p_{k}\right)\left(r e, O^{i}\right)= \begin{cases}t\left(O_{r e}^{i}, S p_{k}\left(O_{r e}^{*}\right)\right) & \text { condmax } \\ 0 & \text { otherwise }\end{cases}$

where $O_{r e}^{i}$ is the conjunction via t of the memberships of all the objects in $O^{i}$. Also

$$
O_{r e}^{*}\left(o_{j}\right)=\frac{O_{r e}\left(o_{j}\right)}{\max _{o \in O} O_{r e}(o)}
$$

and condmax holds iff $\forall o \in O \backslash O^{i}, o^{\prime} \in O^{i}$ it is $O_{r e}(o) \leq O_{r e}\left(o^{\prime}\right)$.

\section{Examples}

Fig. 1 shows an scene containing several objects, that have been numbered for easier identification. Table 1 shows several fuzzy properties for objects in the scene, and the corresponding fulfilment degrees.

Fig. 2 shows four sets of three objects $O^{i}$, marked with an " $x$ " in each case, intended to be referred to by referring expressions "The dark triangles" (Fig. 2(a)), "The circles" (Fig. 2(b)), "The objects at the top-right" (Fig. 2(c)), and "The dark squares" (Fig. 2(d)). Table 2 shows the referential success obtained using Def. 3.1 with the three $k$-specificity measures of Eqs. (6) to (8) and minimum and product as tnorms. The best behaviour is that of measures $C_{\Lambda, 3}$ and $C_{L, 3}$, since they yield the expected values 1 and 0 for the first two cases, and reasonable intermediate values in the other two cases. Measure $C_{3}^{f}$, as discussed in (Marín et al., 2017b) for its corresponding specificity measure, is more suitable for ranking intermediate results when generating the referring expression by means of Greedy approaches.

\section{Conclusions}

The measures proposed are to be employed in REG algorithms for set referring expressions with fuzzy properties. We are about to begin testing which measures are more suitable for providing good results in such setting, in order to validate the proposal.

\section{Acknowledgments}

This work has been partially supported by the Spanish Ministry of Economy and Competitiveness and the European Regional Development Fund - ERDF (Fondo Europeo de Desarrollo Regional - FEDER) under project TIN2014-58227-P Descripción lingüística de información visual mediante técnicas de minería de datos y computación flexible. 


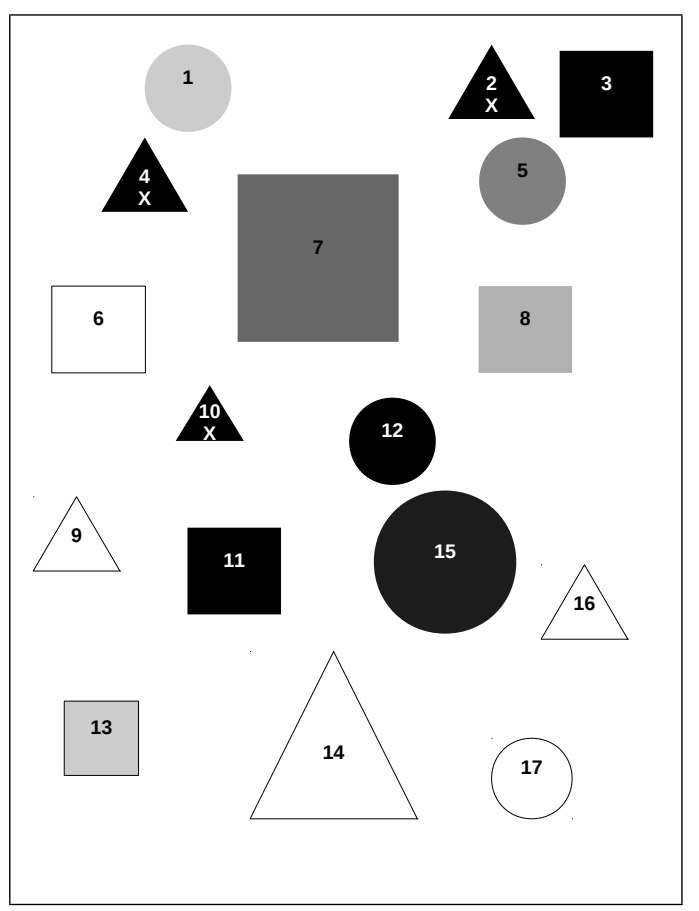

(a) "The dark triangles" matches the marked objects.

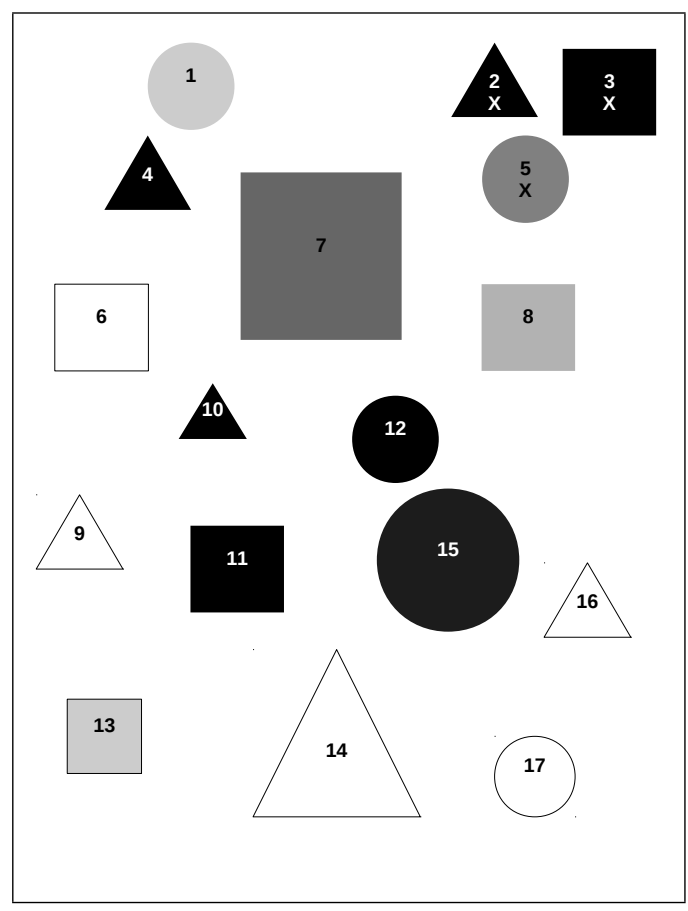

(c) "The objects at the top right" matches the marked objects to a certain degree because of object 8 .

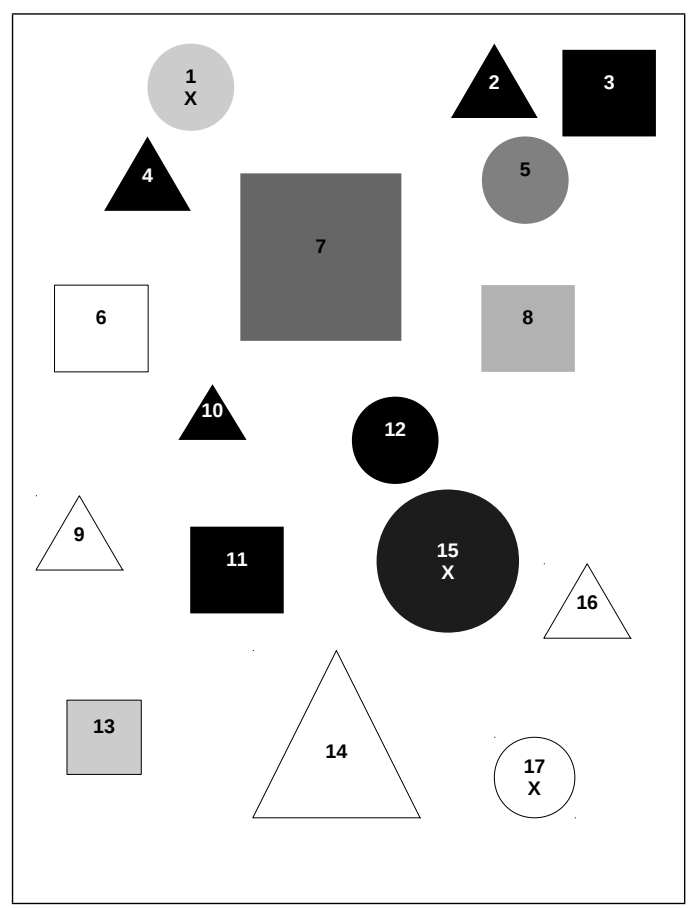

(b) "The circles" does not match the marked objects (also refers to objects 5 and 12).

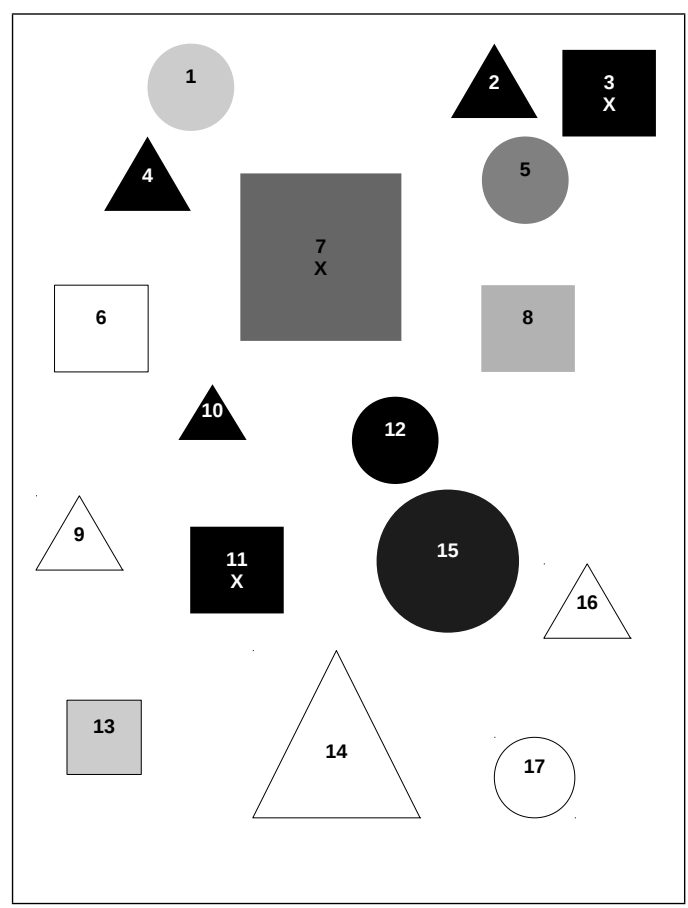

(d) "The dark squares" matches the marked objects to a certain degree because of objects 8 and 13 .

Figure 2: Example scenes and (some valid, some not) expressions. 


\section{References}

L. Garmendia, R. R. Yager, E. Trillas, and A. Salvador. 2003. On t-norms based specificity measures. Fuzzy Sets and Systems, 133(2):237-248.

L. Garmendia, R. R. Yager, E. Trillas, and A. Salvador. 2006. Measures of specificity of fuzzy sets under t-indistinguishabilities. IEEE Transactions on Fuzzy Systems, 14(4):568-572.

A. Gatt, N. Marín, F. Portet, and D. Sánchez. 2016. The role of graduality for referring expression generation in visual scenes. In Proceedings IPMU 2016, Part I, CCIS 610, pages 191-203.

E. Krahmer and K. van Deemter. 2012. Computational generation of referring expressions: A survey. Computational Linguistics, 38(1):173-218.

N. Marín, G. Rivas-Gervilla, and D. Sánchez. 2016. Using specificity to measure referential success in referring expressions with fuzzy properties. In Proceedings FUZZ-IEEE 2016, pages 563-570.

N. Marín, G. Rivas-Gervilla, and D. Sánchez. 2017a. Using measures of k-specificity for the management of count restrictions in flexible querying. In Proceedings SUM 2017.

N. Marín, G. Rivas-Gervilla, D. Sánchez, and R. R. Yager. 2017b. On families of bounded specificity measures. In IEEE International Conference on Fuzzy Systems, FUZZ-IEEE Naples, Italy.

N. Marín, G. Rivas-Gervilla, D. Sánchez, and R. R. Yager. 2017c. Specificity measures and referential success. IEEE Transactions on Fuzzy Systems, In press. DOI: 10.1109/TFUZZ.2017.2694803.

J. Luis González Sánchez, R. González del Campo, and L. Garmendia. 2016. Some new measures of kspecificity. In Advances in Artificial Intelligence - 17th Conference of the Spanish Association for Artificial Intelligence, CAEPIA 2016, Salamanca, Spain, September 14-16, 2016. Proceedings, pages 489-497.

K. van Deemter. 2012. Not Exactly: In Praise of Vagueness. Oxford University Press, Inc., New York, NY, USA.

K. van Deemter. 2016. Computational Models of Referring: A Study in Cognitive Science. MIT Press.

R. R. Yager. 1982. Measuring tranquility and anxiety in decision-making: An application of fuzzy sets. Internat. J. Gen. Systems, 8:139-146.

R. R. Yager. 1990. Ordinal measures of specificity. Internat. J. Gen. Systems, 17:57-72.

R. R. Yager. 1992. On the specificity of a possibility distribution. Fuzzy Sets and Systems, 50:279-292. 